\title{
Increased sleep need and reduction of tuberomammillary histamine neurons after rodent traumatic brain injury.
}

\author{
Daniela Noain ${ }^{1}$, PhD, Fabian Büchele ${ }^{1}$, MD, Sebastian R. Schreglmann ${ }^{1}$, MD, Philipp O. \\ Valko $^{1}$, MD, Yuri V. Gavrilov ${ }^{1}$, MD, Marta M. Morawska ${ }^{1}$, PhD, Lukas L. Imbach ${ }^{1}$, MD, and \\ Christian R. Baumann ${ }^{1}$, MD, Prof.
}

1. Department of Neurology, University Hospital of Zurich, University of Zurich, Zurich, Switzerland.

Running title:

Pleiosomnia and histaminergic loss after rat TBI

Table of Contents title:

Pleiosomnia and histaminergic loss after rat TBI 


\begin{tabular}{|c|c|}
\hline \multirow[b]{2}{*}{ 1. Daniela Noain, PhD (Corresponding author) } & 5. Yuri V. Gavrilov, MD \\
\hline & Department of Neurology \\
\hline Department of Neurology & University Hospital Zurich \\
\hline University Hospital Zurich & Frauenklinikstrasse 26 \\
\hline Frauenklinikstrasse 26 & CH-8091 Zurich \\
\hline \multirow{2}{*}{$\mathrm{CH}-8091$ Zurich } & Switzerland \\
\hline & Tel. 41442555511 \\
\hline Switzerland & Fax 41442554380 \\
\hline Tel. 41442555511 & yury-doctor@mail.ru \\
\hline
\end{tabular}

Fax 41442554380

daniela.noain@usz.ch

\section{Fabian Büchele, MD}

6. Marta M. Morawska, PhD

Department of Neurology

Department of Neurology

University Hospital Zurich

University Hospital Zurich

Frauenklinikstrasse 26

Frauenklinikstrasse 26

CH-8091 Zurich

Switzerland

CH-8091 Zurich

Tel. 41442555511

Switzerland

Fax 41442554380

Tel. 41442555511

marta.morawska@usz.ch

Fax 41442554380

fabian.buechele@usz.ch 
3. Sebastian R. Schreglmann, MD

Department of Neurology

University Hospital Zurich

Frauenklinikstrasse 26

$\mathrm{CH}-8091$ Zurich

Switzerland

Tel. 41442555511

Fax 41442554380

sebastian.schreglmann@googlemail.com

4. Philipp O. Valko, MD

Department of Neurology

University Hospital Zurich

Frauenklinikstrasse 26

$\mathrm{CH}-8091$ Zurich

Switzerland

Tel. 41442555511

Fax 41442554380

philipp.valko@usz.ch
7. Lukas L. Imbach, MD

Department of Neurology

University Hospital Zurich

Frauenklinikstrasse 26

$\mathrm{CH}-8091$ Zurich

Switzerland

Tel. 41442555511

Fax 41442554380

lukas.imbach@usz.ch

8. Christian R. Baumann, MD, Prof.

Department of Neurology

University Hospital Zurich

Frauenklinikstrasse 26

$\mathrm{CH}-8091$ Zurich

Switzerland

Tel. 41442555511

Fax 41442554380

christian.baumann@usz.ch 


\section{Abstract}

Although sleep-wake disturbances are prevalent and well-described after traumatic brain injury, their pathophysiology remains unclear, most likely because human traumatic brain injury is a highly heterogeneous entity which makes the systematic study of sleep-wake disturbances in relation to trauma-induced histological changes a challenging task. Despite increasing interest, specific and effective treatment strategies for posttraumatic sleepwake disturbances are still missing. With the present work, therefore, we aimed at studying acute and chronic sleep-wake disturbances by electrophysiological means, and at assessing their histological correlates after closed diffuse traumatic brain injury in rats with the ultimate goal of generating a model of posttraumatic sleep-wake disturbances and associated histopathological findings that accurately represents the human condition. We assessed sleep-wake behavior by means of standard electrophysiological recordings before and 1,7 , and 28 days after sham or traumatic brain injury procedures. Sleep-wake findings were then correlated to immunohistochemically labeled and stereologically quantified neuronal arousal systems. Compared to control animals, we found that closed diffuse traumatic brain injury caused increased sleep need 1 month after trauma, and sleep was more consolidated. As histological correlate, we found a reduced number of histamine immunoreactive cells in the tuberomammillary nucleus, potentially related to increased neuroinflammation. However, monoaminergic and hypocretinergic neurotransmitter systems in hypothalamus and rostral brainstem were not affected. These results suggest that our rat traumatic brain injury model reflects human posttraumatic sleep-wake disturbances and associated histopathological findings very accurately, thus providing a study platform for novel treatment strategies for affected patients.

\section{Keywords}

Traumatic brain injury; sleep-wake disturbances; tuberomammillary nucleus; histamine; orexin/hypocretin; sleep-wake centers; posttraumatic pleiosomnia; rat model of sleepwake disturbances. 

Introduction

Although traumatic brain injury (TBI) is a very common problem in all populations, the chronic posttraumatic sleep-wake sequelae have been neglected for long, making them a silent epidemic. Only recently, there is increased attention from trauma specialists, neurologists, and psychologists. ${ }^{1-6}$ In light of new evidence, underestimation of sleep-wake disturbances following $\mathrm{TBI}$ by patients themselves may have contributed to this remarkable neglect. ${ }^{4,5}$ Among the most commonly reported posttraumatic SWD are excessive daytime sleepiness (EDS) and excessive sleep need per 24 hours. ${ }^{7,8}$. For the sake of clarity - the term "hypersomnia" has been used for both EDS and excessive sleep need we recently introduced the term "posttraumatic pleiosomnia" for excessive sleep need per 24 hours following TBI. ${ }^{7}$ In autopsy samples of deceased TBI victims, we found a $>40 \%$ loss of wake-maintaining histamine neurons in the tuberomammillary nucleus, i.e. in the posterior hypothalamus, and a more moderate damage to other sleep-wake regulating hypothalamic and brainstem nuclei. ${ }^{9,10}$ Because human $T B I$ is heterogeneous with reference to type, localization, severity of trauma and to the brain it is affecting, it is difficult to systematically study specific alterations in sleep electroencephalography acutely after TBI, and to compare findings to neuropathological outcomes.

In rodents, on the other hand, the effects of reproducible brain lesions on sleepwake behavior and histological outcomes can be studied more systematically. Although decades of animal studies on TBI have thoroughly investigated cognitive performance, neuropsychiatric outcome and motor skills after trauma, ${ }^{11-14}$ there are only a few systematic reports on posttraumatic SWD in rodents. ${ }^{15-22}$ Most of these studies examined sleep-wake behavior only within the first few days after TBI, ${ }^{15,16,19,20}$ while human posttraumatic SWD persist up to months and years. ${ }^{4,5,23,24}$ Sleep-wake outcomes up to 5 weeks after mouse or rat fluid percussion or closed cortical impact TBI have been analyzed recently, but electroencephalographic sleep assessments were not performed ${ }^{18,21}$ or baseline sleep-wake levels were not analyzed. ${ }^{17}$ Willie and colleagues observed by intracerebral microdialysis an abnormal dynamics of hypocretin (orexin) signaling in 
association with reduced wakefulness 3 days after murine TBI. ${ }^{15}$ Similarly, Lim and colleagues reported that mild TBI in mice causes a persistent inability to maintain wakefulness and decreases arousal-promoting hypothalamic hypocretin neuron activation during wakefulness. ${ }^{16}$ Skopin et al also observed the appearance of chronic increased sleep in relation to a significant loss of hypocretin cells after lateral fluid percussion, while posttraumatic changes in other neurotransmitter systems, such as the highly affected histaminergic one, ${ }^{9}$ were not explored. ${ }^{22}$ Up to date, thus, there is no study to explore changes in short- and long-term posttraumatic sleep-wake behavior compared to baseline recordings and in relation to the integrity of multiple sleep-wake modulating neurotransmitter systems. Such knowledge should add validity to a rodent TBI model as representative of the human condition and, thus, offer novel windows of opportunity to develop treatments for affected TBI patients.

With this study, we aimed at investigating sleep-wake behavior after TBI in rats, and to examine whether the integrity of sleep-wake modulating neurotransmitter systems in hypothalamus and brainstem is disturbed in association with sleep-wake findings.

\section{Materials and methods}

\section{Animals}

We used adult male Sprague-Dawley rats (Harlan Laboratories Inc, NL) weighing 230-270 g ( 8-9 weeks of age) at arrival for all experiments. The rats were housed in groups for 2-3 days prior to electroencephalograpy/electromyography (EEG/EMG) electrode implantations and afterwards single-caged with ad libitum access to food and water. The animal room temperature was constantly maintained at $21-23^{\circ} \mathrm{C}$, and animals were kept under a $12 \mathrm{~h}$ light-dark cycle starting at 8.00 or 9.00 a.m., according to season. All experiments were approved by the veterinary office of the Canton Zurich and conducted according to the local guidelines for care and use of laboratory animals. 
Surgeries

We performed all surgical procedures under deep anesthesia with isoflurane $(4.5 \%$ for induction, $2.5 \%$ for maintenance). We administered buprenorphine $(0.05 \mathrm{mg} / \mathrm{kg}$, s.c.) for analgesia. Over the first week after surgeries, we monitored all animals on a daily basis and weekly thereafter, by assessing weight progression and wound healing.

\section{EEG/EMG electrodes implantation}

We implanted rats with EEG/EMG electrodes for recording of vigilance states as described earlier. ${ }^{25}$ Briefly, we inserted 4 gold-plated miniature screws, one pair for each hemisphere, bilaterally into the rats' skull following specific stereotaxic coordinates: the anterior electrodes were implanted $3 \mathrm{~mm}$ posterior to bregma and $2 \mathrm{~mm}$ lateral to the midline, and the posterior electrodes $6 \mathrm{~mm}$ posterior to bregma and $2 \mathrm{~mm}$ lateral to the midline. We left the skull above the frontal cortex unimplanted for TBI impact. For the monitoring of muscle tone, a pair of gold wire inserted into the rats' neck muscle served as EMG electrodes. All electrodes were connected to a head connector (Farnell AG, Switzerland) and fixed to the skull with dental cement. A recovery of 8 to 10 days was granted to all animals before further interventions.

\section{Traumatic brain injury}

This novel TBI model was introduced earlier. ${ }^{25}$ Briefly, after 8-10 days of recovery, the EEG/EMG implanted rats weighed 300-350 g ( 10-11 weeks old) and underwent closed skull TBI in the prefrontocortical area of the brain using a closed diffuse TBI-inducing device (Figure 1a). Rats under deep anesthesia received a $0.5-0.7 \mathrm{~cm}$ scalp incision over the midline in the prefrontocortical area of the brain, thus anterior to the implanted electrodes. For TBI, using a system of sliding rings, we released a $2500 \mathrm{~g}$ stainless steel rod provided with a flat silicon tip adjusted to an angle of 70 and falling from a height of 25 $\mathrm{cm}$ over the exposed skull. The latter was covered by a $1 \mathrm{~mm}$ thick metal plate to prevent bone fractures. Thereafter, we closed and disinfected the skin, and animals returned to 
their home cage and were monitored continuously for at least 1 hour or until full recovery from sedation and normal home cage behavior were observed. Analgesia was provided using buprenorphine. SHAM procedures were identical to TBI ones, except the rod was not released to its intended fall. As reported earlier, ${ }^{25}$ despite memory function was impaired, rats survived TBI without major neurological or motor deficits. EEG/EMG implants proved stable for at least 1 month and enabled qualitative and quantitative sleep analyses. Histologically, we observed no major bleedings or necrosis but intense diffuse axonal damage after TBI.

\section{EEG/EMG recording and scoring}

We conducted bilateral 24h EEG/EMG recordings one day prior to injury to serve as baseline, as well as 1, 7 and 28 days after trauma (Figure 1b). For this purpose, we transferred animals to special recording cages with food and water available ad libitum, where they had an adaptation period of two days before recordings took place. During every recording session, we recorded SHAM and TBI rats simultaneously. For analysis purposes, we divided the $24 \mathrm{~h}$ scoring sessions into $12 \mathrm{~h}$ light and dark periods (Figure 1b), and assessed the proportion of each vigilance state separately for each period. As before, based on EEG/EMG patterns, we assessed three behavioral states: non-rapid eye movement (NREM) sleep, rapid eye movement (REM) sleep and wakefulness (WAKE) (Figure 1C). ${ }^{25}$ Sleep-wake proportion results from the light period were previously reported. ${ }^{25}$

In order to further explore the characteristics of sleep in the dark period 28 days after TBI, we calculated the index of sleep fragmentation as introduced before. ${ }^{4}$ Briefly, sleep fragmentation is defined as the number of sleep bouts divided by the total number of epochs in the same sleep stage. The sleep fragmentation index reflects, therefore, the relative number of behavioural state bouts per time (e.g. highly fragmented sleep with many changes in sleep-wake behavioural states will result in a high sleep fragmentation index). Finally, we determined the delta power $(1-4 \mathrm{~Hz})$ of NREM sleep at baseline, and 1, 7 and 28 days after both TBI and SHAM procedures to assess whether TBI caused changes in 
sleep depth as compared to SHAM controls. TBI effect 28 days after trauma was calculated as $\triangle \mathrm{TBI}$-SHAM\% per $24 \mathrm{~h}$ in 2 hour bins during both dark and light periods.

\section{Immunohistochemistry}

Rats were sacrificed 30 days after trauma by classic cardiac perfusion under pentobarbital anesthesia $(75 \mathrm{mg} / \mathrm{kg}$ ). We dissected brains, post-fixed and dehydrated them in $4 \%$ PFA/15\% sucrose (Sigma-Aldrich) in PBS at 4 을 overnight. A further dehydration step in $30 \%$ sucrose in PBS followed. After complete dehydration, we froze brains in dry ice and stored them at $-80 \stackrel{\circ}{C}$ until further use.

To assess posttraumatic histological outcomes in relation to sleep-wake behavior, we explored 4 sleep-wake regulating neurotransmitter nuclei in the hypothalamus and brainstem. We incubated free-floating $40 \mu \mathrm{m}$ coronal brain cryostat sections at $4 \stackrel{\circ}{ }{ }^{\circ}$ with agitation with primary antibodies against: a) histamine (HA, rabbit anti-HA, 1:100, Millipore, cat\#AB5885, 7 days incubation); b) melanin-concentrating hormone $(\mathrm{MCH}$, rabbit anti-MCH, 1:10,000, Phoenix Europe $\mathrm{GmbH}, \mathrm{H}-070-47,16$ hours incubation); c) hypocretin/orexin (Hcrt-1, goat anti-hcrt1 [C19], 1:5,000, Santa Cruz; sc-8070, 16 hours incubation); and d) tyrosine hydroxylase (TH, mouse anti-TH, 1:8,000, Millipore, MAB318, 16 hours incubation), a marker of catecholaminergic cells. After primary antibody incubation, the sections were washed and then incubated in the corresponding secondary antibodies: biotinylated goat anti-rabbit secondary antiserum (1:250; VectorLabs), biotinylated horse anti-goat secondary antiserum (1:600, VectorLabs) or biotinylated goat anti-mouse secondary antiserum (1:500; Santa Cruz Biotechnology) for 2 hours at room temperature. Then, the sections were incubated with $A B C$ complex (1:100, VectorLabs) and exposed to $0.025 \%$ DAB in TBS in the presence of $0.05-0.1 \% \mathrm{H}_{2} \mathrm{O}_{2}$ to produce dark brown precipitates and finally mounted. All results were compared to SHAM controls.

In the TMN, the only nucleus in which we observed significant loss of neurotransmitter immunoreactivity, we examined by double immunofluorescence the association between such loss and other histological sequelae of TBI. To this end, we co-incubated TMN hypothalamic sections with anti-histidine decarboxylase, HDC -a marker of histaminergic 
cells- (rabbit anti-HDC, 1:5000, American Research Products, CAT\#03-16045) and one of the following antibodies: anti-amyloid precursor protein-1, APP-1 -a marker of diffuse axonal injury- (mouse anti-APP-1, 1:300, Millipore, MAB348); anti-Ox42 -a neuroinflammation marker- (mouse anti-Ox42/anti- CD11b/c, 1:500, Abcam, ab1211); anti-glial fibrillary acidic protein, GFAP - a marker of astrogliosis (mouse anti-GFAP, coubled with Cy3, 1:400, Sigma-Aldrich, C9205). After primary antibody incubation overnight, the sections were washed and then incubated in the corresponding secondary antibodies: goat anti-rabbit Alexa 488 (Molecular Probes, 1:500) and donkey anti-mouse Alexa 568 (Molcular Probes, 1:500). After washings, the fluorescent sections were mounted (Aquamount, Fischer sci), coverslipped and stored in the dark at 4 o $\mathrm{C}$ until microscopy for qualitative evaluation.

\section{Stereological counting}

We quantified hypothalamic and brainstem neuronal populations by blinded semiautomatic stereological countings using the optical fractionator method on a Zeiss Axio Imager M2 microscope (Karl Zeiss, Jena, Germany) equipped with a Ludl MAC 6000 stage (Ludl Electronic Products, Hawthorne, NY) and MicroBrightField Stereo Investigator 10.5 software (MBF Bioscience, Williston, VT). We used a 2.5x objective for outlining brain areas with immunopositive cells, and a 40x oil immersion objective (1.3 numerical aperture) for stereological counting. We defined the borders of the reference space by staining pattern. Only animals with a complete set of undamaged sections were included in the final analysis. We optimized counting frames and sampling grid sizes for each antigen to achieve a Gundersen coefficient of error $(\mathrm{CE})<0.08$. A guard distance of $2.0 \mu \mathrm{m}$ was used during cell counting to avoid introduction of errors due to sectioning artifacts.

\section{Statistical analyses}

Two-tailed, paired and unpaired t-tests with or without Bonferroni corrections, repeated measures ANOVA, post hoc analysis of Fisher (LSD), ANCOVA and Pearson's correlations 
were performed as indicated in the results section and figure legends. All bars in the graphs represent the groups' mean and the error bars indicate standard error of mean (S.E.M.). The sample size is indicated in each figure legend as well as the statistics $P$ value. Significant differences were acknowledged only to $P$ values lower than 0.05 . Thus, the performed analyses provide a margin of confidence of $95 \%$ to our results.

\section{Results}

Increased sleep need 4 weeks after closed diffuse TBI in rodents

As previously reported ${ }^{25}$, sleep-wake proportions in the light period (rats' resting phase) were unchanged in TBI compared to SHAM animals. However, analysis of dark period (rats' active phase) EEG signals revealed an increase of NREM sleep in injured rats 28 days after TBI, as evidenced by the significant decrease of WAKE (Figure $2 a$, SHAM $(n=6)$ vs. TBI $(n=7)$, ${ }^{*} \mathrm{P}<0.05$, two-tailed unpaired $t$ test with Bonferroni correction) and increase of NREM sleep (Figure $2 \mathbf{b}$, SHAM $(n=6)$ vs. TBI $(n=7),{ }^{*} P<0.05$, two-tailed unpaired t test with Bonferroni correction) compared to SHAM rats. Differences of WAKE and NREM sleep proportions between SHAM and TBI groups were not observed at any other time point analyzed in the dark period (BL, P1, P7; Figure 2a, b). REM sleep was unaltered after TBI for all time points (Figure 2c).

To gain more detailed insights into the 24 hours-time course of vigilance state proportions 28 days after TBI, we analyzed the recordings of both SHAM $(n=6)$ and TBI $(n=7)$ animals in 2-hour time bins. This analysis revealed that TBI animals had intermittent periods of increased sleep need at the cost of wakefulness between the $3^{\text {rd }}$ and $5^{\text {th }}$ hour (WAKE: ${ }^{*} \mathrm{P}<0.05$; NREM: ${ }^{*} \mathrm{P}=0.06$, repeated measures ANOVA, post hoc analysis of Fisher) and the $9^{\text {th }}$ and $11^{\text {th }}$ hour (WAKE and NREM: ${ }^{*} \mathrm{P}<0.05$, repeated measures ANOVA, post hoc analysis of Fisher) during the dark period (Figure $\mathbf{2 d}$ ). Again, the distribution of REM sleep throughout the 24 hours period was similar between both groups (Figure $\mathbf{2 d}$ ). 
To further determine the existence of posttraumatic pleiosomnia, we calculated the cumulated total sleep (TS, NREM+REM) per 24 hours and in separate dark and light periods (Figure 3) in TBI animals as compared to SHAM controls. Total sleep was increased both in the dark period (Figure 3a; SHAM $(n=6)$ vs. TBI $(n=7),{ }^{* *} P<0.005$, one-tailed unpaired $t$ test) and per 24 hours (Figure 3c; ${ }^{*} P<0.05$, SHAM ( $\left.n=6\right)$ vs. TBI $(n=7)$, one-tailed unpaired $t$ test), but unaltered in the light phase (Figure $\mathbf{3 b}$ ).

\section{Sleep is more stable after TBI in rats}

Sleep fragmentation index for NREM sleep 28 days after trauma in TBI animals was reduced when compared to the dark phase baseline value (Figure 4a; ${ }^{*} \mathrm{P}<0.05, \mathrm{BL}$ (SHAM $n=7, T B \mid n=6$ ) vs. P28 (SHAM $n=6, T B \mid n=7$ ), two-tailed paired t test), while we observed unchanged fragmentation indices for WAKE and REM and all states in SHAM animals. The number of WAKE, NREM sleep and REM sleep bouts were invariable over time both in SHAM and TBI groups compared to the respective baselines (Figure 4b). Finally, we evaluated delta EEG power, a measure of deep NREM sleep, per 24 hours at all 4 time points in the TBI and SHAM groups. We observed a $\sim 25 \%$ transient increase of NREM relative delta power in the TBI group compared to SHAM controls at 7 days after trauma, but no permanent long term effect (Figure 4c; group difference $\mathrm{P}=0.008$, linear mixed model ANCOVA, significant effect for $\mathrm{P} 7$ isolated $\left.{ }^{*} \mathrm{P}<0.05\right)$.

Loss of neuronal immunoreactivity in the histaminergic tuberomammillary nucleus after closed diffuse TBI

We performed immunohistochemistry studies in SHAM and TBI brains (Figure 5a). We found that the number of $\mathrm{MCH}, \mathrm{Hcrt}-1$ and noradrenergic TH neurons was unchanged after TBI. However, we observed a significant $\sim 36 \%$ decrease in the number of $\mathrm{HA}$ immunoreactive neurons in TBI brains (Figure 5a; SHAM ( $n=6)$ vs. TBI $(n=7),{ }^{* * *} P<0.001$, two-tailed unpaired t test). Pearson's correlation analyses including all SHAM and TBI animals $(n=13)$ evidenced both positive and negative associations between the number of 
immunopositive HA neurons in the TMN and the amounts of WAKE and NREM sleep, respectively, in the dark period 28 days after TBI (Figure 5b, correlation HA cell number and WAKE time in the dark period: $\mathrm{P}=0.008$, Pearson $\mathrm{r}=0.6931$; correlation $\mathrm{HA}$ cell number and NREM time in the dark period: $\mathrm{P}=0.01$, Pearson $\mathrm{r}=-0.6384$; Pearson's correlation analysis). No significant correlations were found in SHAM $(n=6)$ and TBI $(n=7)$ groups individually (data not shown).

To evaluate the potential mechanisms underlying decreased immunoreactivity of HA neurons, we performed co-localization studies of histidine decarboxylase (HDC) with markers of neuroinflammation (Ox42), astrogliosis (GFAP) and diffuse axonal injury (APP-1) in sections containing the tuberomammillary nucleus (Figure 6a). There, we found increased neuroinflammation around the tuberomammillary nucleus following TBI as compared to SHAM controls (Figure 6b). Conversely, no salient differences were noted as far as astrocytic scarring and diffuse axonal injury are concerned (Figure $6 c$ and $\mathbf{d}$ ). Regarding the latter, there was abundant somatic APP-1 immunoreactivity in colocalization with HDC, but only marginal colocalizing axonal staining, both in SHAM and TBI animals (Figure 6d). This indicates that there is only little axonal accumulation of APP-1 in tuberomammillary neurons, irrespective of the presence of TBI.

\section{Discussion}

In the present study, we provide evidence of increased sleep need one month after closed diffuse TBI in rats, and more consolidated NREM sleep in the dark phase. These findings are strikingly similar to those observed in human TBI patients, ${ }^{4,5}$ but also to a previous rodent TBI study applying the lateral fluid percussion model. ${ }^{22}$ In addition, we observed a $\sim 36 \%$ reduction of histamine immunoreactive neurons in the TMN, and pooled data revealed an inverse correlation between the number of histamine neurons and the amount of NREM sleep in the active dark period, suggesting that this loss might contribute to increased sleep need. Recent results in human autopsy material have suggested that TBI - which is very heterogeneous in human patients - causes a widespread loss of 
hypothalamic cells, particularly a $>40 \%$ reduction of histaminergic neurons in the TMN of the posterior hypothalamus, and a less distinct damage to other hypothalamic and brainstem populations. ${ }^{9,10}$

In an attempt to elucidate whether the observed increased NREM sleep reflects a higher consolidation of behavioral states, or whether it is associated with many short episodes of NREM sleep which would indicate acquired sleep instability, we determined the number of sleep bouts in the dark phase of TBI rats 28 days after trauma and it was similar to the one assessed at baseline. However, the average length of NREM bouts was increased, yielding a reduced fragmentation index. Thus, these results suggest that TBI induces a sleep-wake pattern of more consolidated sleep rather than a fragmented one, once more in agreement with our previous observations in patients ${ }^{4}$ but in contrast with previous rodent reports. ${ }^{22}$ The coincidence of present results with the human literature supports the notion that our TBI model reproduces human TBI and posttraumatic SWD in a very accurate manner. Interestingly, long sleep bouts are more likely to reflect excessive sleep need (i.e. pleiosomnia) than excessive day-time sleepiness (EDS) which is characterized by frequently intruding short sleep bouts, reflecting increased sleep pressure during wakefulness. ${ }^{26,27}$

Again in agreement with human findings, ${ }^{4}$ we only observed a rather mild and transient increase of delta power 7 days after TBI, indicating that posttraumatic pleiosomnia is characterized by increased, consolidated sleep, but not by enhanced slow-wave activity which would indicate chronic deeper sleep. TMN neurons are proposed to have a wakefulness-maintenance function. ${ }^{28}$ Thus, their loss or impairment could potentially cause or contribute to pleiosomnia, in line with the results of the present study.

We observed increased sleep need in TBI rats compared to SHAM animals only 28 days after trauma and not at earlier time points. This might be accounted for by two main reasons. First, unspecific stress reactions and higher anxiety levels could predominantly impact sleep-wake behavior within 1 and 7 days after trauma. In this line, a marked and 
transient increase of NREM sleep with decreased WAKE time was observed in both SHAM and TBI animals 1 day after surgery. This might reflect an unspecific "surgery effect" related to surgical-anesthesia and/or post-surgical analgesia, as well as the general need of recovery acutely after the interventions. At 7 days after surgery, animals appear to have recovered normal sleep-wake behavior, as evidenced by vigilance time proportions similar to baseline levels, but anxiety levels might still be higher than normal. We have not performed corresponding anxiety measuring tests in our study, which must be noted as a limitation and should be performed in future approaches. Second, the late occurrence of increase sleep need could be linked to a slow development of persistent and long-term trauma-induced damage to sleep-wake regulating neuronal populations. Long-term secondary injury after trauma has been studied in depth and nowadays, posttraumatic injury is rather seen as a disease process than as a single event. ${ }^{29-31}$ For instance, it is permanent and caused by non-reversible pathological alterations, which fits with the current definition of the World Health Organization for chronic disease. ${ }^{31}$

The distinct vulnerability of histaminergic neurons compared to other hypothalamic populations might be explained by their exposed anatomical position neighboring the base of the skull, both in humans and rodents. Thus, acceleration/deceleration mechanisms of the brain within the skull might cause direct superficial injuries in addition to tension and compression mechanisms in this area, ultimately leading to damage of the affected neurons. At the time point of study termination, we did neither observed APP-1 accumulation in axons in the TMN nor enhanced gliosis around the TMN. This may indicate that the processes leading to diminished HA cell counts are others than APP-mediated degeneration or excessive scarring. On the other hand, we cannot exclude the possibility that the latter might play a role at earlier posttraumatic time points, and studies with longitudinal histological evaluations would be necessary for clarification. However, the observation that sleep-wake disturbances only appear with significant delay after trauma rather suggests more enduring mechanisms of action. As a possible explanation, we found enhanced neuroinflammation around the TMN following TBI. As the primary immune cells in the brain, microglia are known to release numerous factors, including pro-inflammatory 
and anti-inflammatory cytokines, chemokines, nitric oxides, prostaglandins, growth factors, and superoxide species, which could modulate secondary injury, also over a certain time span. ${ }^{32}$ Yet, it remains unclear if the findings of reduced histaminergic cell counts and increased neuroinflammation are only coincidental or also causally related. Thus, further studies including a more detailed characterization of the inflammatory response and anti-inflammatory treatment are warranted to answer this question. Overall, consecutive follow up studies are due to determine the exact nature and progression of the found histopathological changes after rat TBI and, moreover, determine their causal relationship with the observed altered sleep-wake phenotype after trauma.

Furthermore, the more restricted impairment observed in rodent tissue as compared to human brains, might well respond to a milder severity of trauma achieved in our animal model of closed TBI, which possibly does not reflect as high and uncontrolled trauma severity as observed in fatal human TBI cases. ${ }^{9,10}$ For instance, our TBI model is a closed skull model with $100 \%$ survival rate and no long-term motor impairments, ${ }^{25}$ which may well represent TBI in survivors with posttraumatic sleep-wake disturbances, but probably does not represent fatal human TBI cases. Furthermore, our trauma induction allows a sensible amount of head movement in the vertical axis but, for the sake of reproducibility, there are almost no lateral movements during trauma induction due to a partial head fixation system, which may also account for a milder trauma outcome by reducing the number of angles in which the subjects' head can move towards and thus, damage the brain.

Altogether, our study reports long-term posttraumatic sleep-wake disturbances with consolidated, excessive sleep need in association with reduced histaminergic immunoreactivity, possibly related to trauma-induced neuroinflammation. Due to its resemblance with the human condition, this rodent model could allow further studies and lead to novel and tailored therapies for posttraumatic pleiosomnia - a symptom that, so far, cannot be treated. 
DN and CRB designed the experiments. DN, FB, SRS, PV, YG, and MMM executed the experiments. DN, FB, SRS, PV, YG and LLI analyzed data. DN and FB prepared the figures. $\mathrm{DN}, \mathrm{FB}, \mathrm{PV}$ and $\mathrm{CRB}$ wrote the manuscript. All authors revised and edited the manuscript. The authors would like to thank Tom Scammell for his constructive criticisms and Ertugrul Cam, Aleksandra Hodor, Amandine Valomon and Evangelia Symeonidou for their helpful assistance.

This project was supported by the Swiss National Science Foundation (SNSF, grant no. 125504) and by the Clinical Research Priority Program "Sleep and Health" of the University of Zurich.

\section{Authors' disclosures}

All authors declare no competing or financial conflicts. 


\section{References}

1. Rao, V., Rollings, P. (2002). Sleep Disturbances Following Traumatic Brain Injury. Curr Treat. Options Neurol. 4, 77-87.

2. Cohen, M., Oksenberg, A., Snir, D., Stern, M.J., Groswasser, Z. (1992). Temporally related changes of sleep complaints in traumatic brain injured patients. J. Neurol. Neurosurg. Psychiatry 55, 313-315.

3. Castriotta, R.J., Wilde, M.C., Lai, J.M., Atanasov, S., Masel, B.E., Kuna, S.T. (2007). Prevalence and consequences of sleep disorders in traumatic brain injury. J. Clin. Sleep Med. 3, 349-356.

4. Imbach, L.L., Valko, P.O., Li, T., Maric, A., Symeonidou, E.R., Stover, J.F., Bassetti, C.L., Mica, L., Werth, E., Baumann, C.R. (2015). Increased sleep need and daytime sleepiness 6 months after traumatic brain injury: a prospective controlled clinical trial. Brain 138, 726735.

5. Imbach, L.L., Büchele, F., Valko, P.O., Li, T., Maric, A., Stover, J.F., Bassetti, C.L., Mica, L., Werth, E., Baumann, C.R. (2016). Sleep-wake disorders persist 18 months after traumatic brain injury but remain underrecognized. Neurology 86, 1945-1949.

6. Ouellet, M.C., Beaulieu-Bonneau, S., Morin, C.M. (2015). Sleep-wake disturbances after traumatic brain injury. Lancet Neurol. 14, 746-757.

7. Sommerauer, M., Valko, P.O., Werth, E., Baumann, C.R. (2013). Excessive sleep need following traumatic brain injury: a case-control study of 36 patients. J. Sleep Res. 22, 634639.

8. Guilleminault, C., Faull, K.F., Miles, L., van den Hoed, J. (1983). Posttraumatic excessive daytime sleepiness: a review of 20 patients. Neurology 33, 1584-1589.

9. Valko, P.O., Gavrilov, Y.V., Yamamoto, M., Finn, K., Reddy, H., Haybaeck, J., Weis, S., Scammell, T.E., Baumann, C.R. (2015). Damage to histaminergic tuberomammillary neurons and other hypothalamic neurons with traumatic brain injury. Ann. Neurol. 77, 177-182. 
10. Valko, P.O., Gavrilov, Y.V., Yamamoto, M., Noain, D., Reddy, H., Haybaeck, J., Weis, S., Baumann, C.R., and Scammell, T.E. (2016). Damage to Arousal-Promoting Brainstem Neurons with Traumatic Brain Injury. Sleep 39, 1249-1252.

11. Gorman, L.K., Shook, B.L., Becker, D.P. (1993). Traumatic brain injury produces impairments in long-term and recent memory. Brain Res. 614, 29-36.

12. Abdel Baki, S.G., Kao, H.Y., Kelemen, E., Fenton, A.A., Bergold, P.J. (2009). A hierarchy of neurobehavioral tasks discriminates between mild and moderate brain injury in rats. Brain Res. 1280, 98-106.

13. Pandey, D.K., Yadav, S.K., Mahesh, R., Rajkumar, R. (2009). Depression-like and anxietylike behavioural aftermaths of impact accelerated traumatic brain injury in rats: a model of comorbid depression and anxiety? Behav. Brain Res. 205, 436-442.

14. Chen, Y., Constantini, S., Trembovler, V., Weinstock, M., Shohami, E. (1996). An experimental model of closed head injury in mice: pathophysiology, histopathology, and cognitive deficits. J. Neurotrauma 13, 557-568.

15. Willie, J.T., Lim, M.M., Bennett, R.E., Azarion, A.A., Schwetye, K.E., Brody, D.L. (2012). Controlled cortical impact traumatic brain injury acutely disrupts wakefulness and extracellular orexin dynamics as determined by intracerebral microdialysis in mice. J. Neurotrauma 29, 1908-1921.

16. Lim, M.M., Elkind, J., Xiong, G., Galante, R., Zhu, J., Zhang, L., Lian, J., Rodin, J., Kuzma, N.N., Pack, A.I., Cohen, A.S. (2013). Dietary therapy mitigates persistent wake deficits caused by mild traumatic brain injury. Sci. Transl. Med. 5, 215 ra173.

17. Petraglia, A.L., Plog, B.A., Dayawansa, S., Chen, M., Dashnaw, M.L., Czerniecka, K., Walker, C.T., Viterise, T., Hyrien, O., Iliff, J.J., Deane, R., Nedergaard, M., Huang, J.H. 2014). The Spectrum of Neurobehavioral Sequelae after Repetitive Mild Traumatic Brain Injury: A Novel Mouse Model of Chronic Traumatic Encephalopathy. J. Neurotrauma 31, 1211-1224.

18. Rowe, R.K., Harrison, J.L., O'Hara, B.F., Lifshitz, J. (2014). Diffuse brain injury does not affect chronic sleep patterns in the mouse. Brain Inj. 28, 504-510. 
19. Rowe, R.K., Striz, M., Bachstetter, A.D., Van Eldik, L.J., Donohue, K.D., O'Hara, B.F., Lifshitz, J. (2014). Diffuse brain injury induces acute post-traumatic sleep. PloS one 9, e82507.

20. Sabir, M., Gaudreault, P.O., Freyburger, M., Massart, R., Blanchet-Cohen, A., Jaber, M., Gosselin, N., Mongrain, V. (2015). Impact of traumatic brain injury on sleep structure, electrocorticographic activity and transcriptome in mice. Brain Behav. Immun. 47, 118-130.

21. Hazra, A., Macolino, C., Elliott, M.B., Chin, J. (2014). Delayed thalamic astrocytosis and disrupted sleep-wake patterns in a preclinical model of traumatic brain injury. J. Neurosci. Res. 92, 1434-1445.

22. Skopin, M.D., Kabadi, S.V., Viechweg, S.S., Mong, J.A., Faden, A.I. (2015). Chronic decrease in wakefulness and disruption of sleep-wake behavior after experimental traumatic brain injury. J. Neurotrauma 32, 289-296.

23. Baumann, C.R., Werth, E., Stocker, R., Ludwig, S., Bassetti, C.L. (2007). Sleep-wake disturbances 6 months after traumatic brain injury: a prospective study. Brain 130, 18731883.

24. Kempf, J., Werth, E., Kaiser, P.R., Bassetti, C.L., Baumann, C.R. (2010). Sleep-wake disturbances 3 years after traumatic brain injury. J. Neurol. Neurosurg. Psychiatry 81, 1402-1405.

25. Büchele, F., Morawska, M.M., Schreglmann, S.R., Penner, M., Muser, M., Baumann, C.R., Noain, D. (2016). Novel rat model of weight drop-induced closed diffuse traumatic brain injury compatible with electrophysiological recordings of vigilance states. J. Neurotrauma 33,1171-1180.

26. Baumann, C.R., Werth, E., Stocker, R., Ludwig, S., Bassetti, C.L. (2007). Sleep-wake disturbances 6 months after traumatic brain injury: a prospective study. Brain 130, 18731883.

27. Verma, A., Anand, V., Verma, N.P. (2007). Sleep disorders in chronic traumatic brain injury. J. Clin. Sleep Med. 3, 357-362. 
29. Bramlett, H.M., Dietrich, W.D. (2002). Quantitative structural changes in white and gray matter 1 year following traumatic brain injury in rats. Acta Neuropathol. 103, 607614.

30. Inglese, M., Makani, S., Johnson, G., Cohen, B.A., Silver, J.A., Gonen, O., Grossman, R.I. (2005). Diffuse axonal injury in mild traumatic brain injury: a diffusion tensor imaging study. J. Neurosurg. 103, 298-303.

31. Masel, B.E., DeWitt, D.S. (2010). Traumatic brain injury: a disease process, not an event. J. Neurotrauma 27, 1529-1540.

32. Jin, Y., Wang, R., Yang, S., Zhang, X., Dai, J. (2017). Role of microglia autophagy in microglia activation after traumatic brain injury. World Neurosurg. 100, 351-360. 
Figure legends

\section{Figure 1}
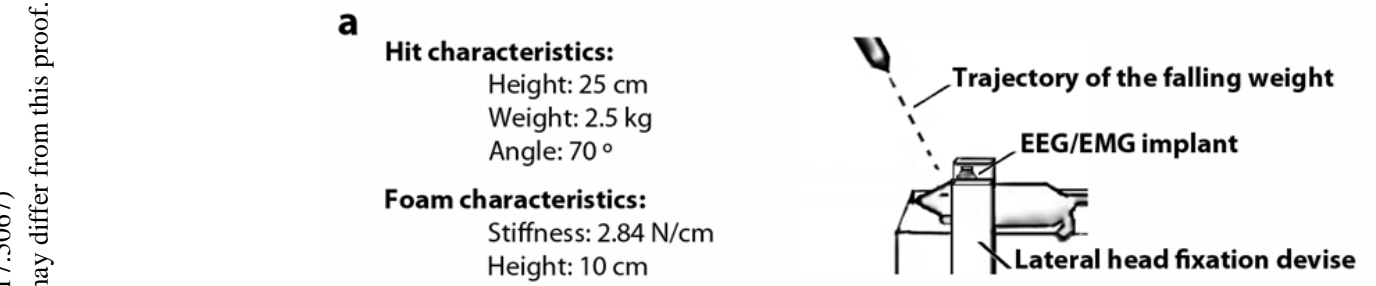

b

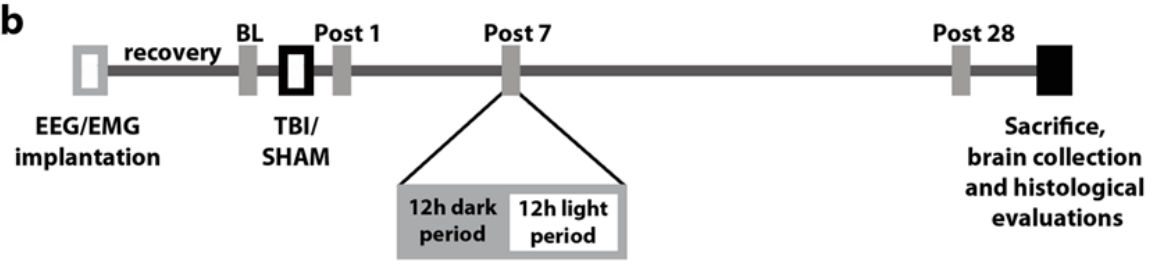

C

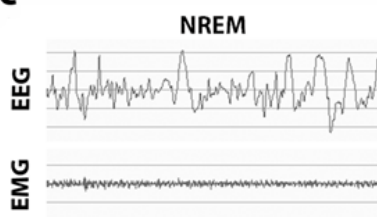

REM

WAKE

Figure 1. Experimental design. a) Information on hit and foam characteristics and schematic representation of our TBI device. b) Timeline: Implantation of EEG/EMG electrodes for the recording of behavioral states followed by recovery for 8-10 days and a baseline 24h EEG/EMG recording 1 day previous to traumatic brain injury (TBI) or SHAM procedures. Posttraumatic EEG/EMG recordings of sleep-wake 1, 7 and 28 days after TBI. Rats were then sacrificed 30 days after SHAM or TBI. Sleep-wake recordings of $24 \mathrm{~h}$ were split into two $12 \mathrm{~h}$ periods, dark and light. c) Assessment of 3 behavioral states: non-rapid eye movement sleep (NREM), rapid eye movement sleep (REM) and wakefulness (WAKE). 


\section{Figure 2}

a

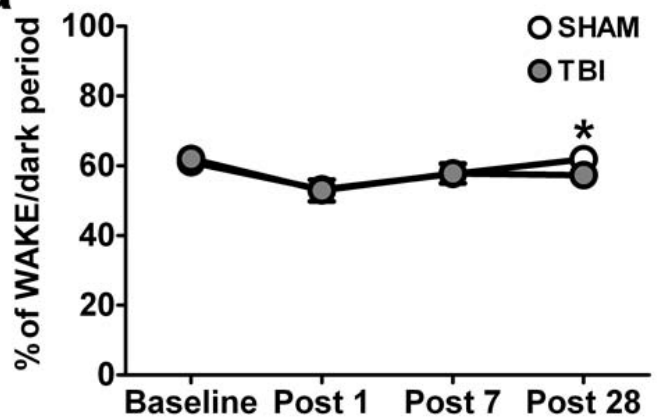

C

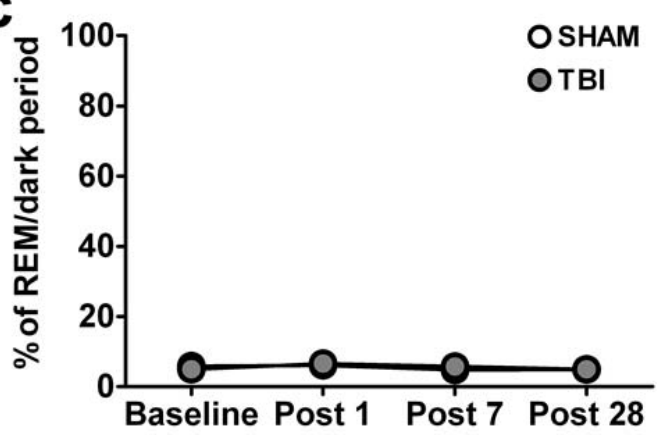

b

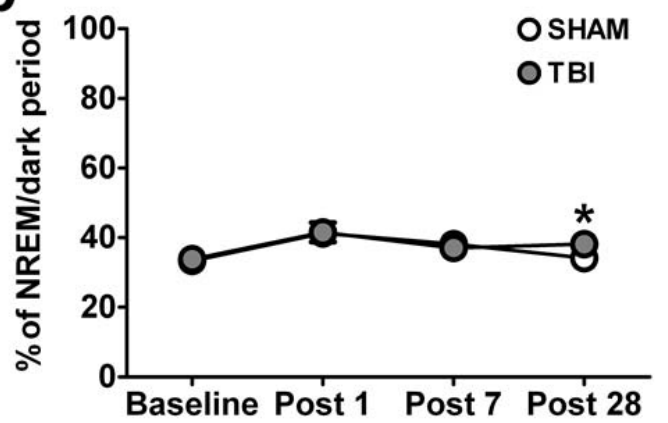

d

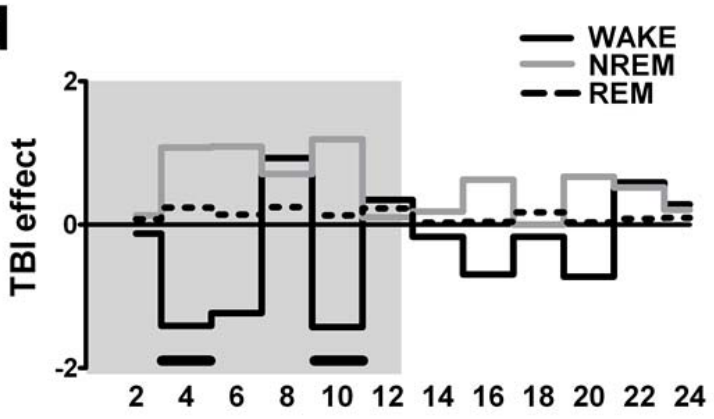

Figure 2. Proportion of behavioral states after traumatic brain injury. Decrease in WAKE (a) and increase NREM sleep (b) in the dark period 28 days after TBI (SHAM vs. TBI, ${ }^{*} \mathrm{P}<0.05$, two-tailed unpaired t test with Bonferroni correction) while no changes in REM sleep (c). Two-hour time bins analysis 28 days after TBI: increased NREM sleep and decreased WAKE mainly at 2 time points during the dark period (d; SHAM vs. TBI: horizontal black bars represent $\mathrm{P}<0.05$, repeated measures ANOVA, post hoc comparisons of Fisher). $\mathrm{N}=5-7$ animals in each group, circles represent mean+/-SEM. 

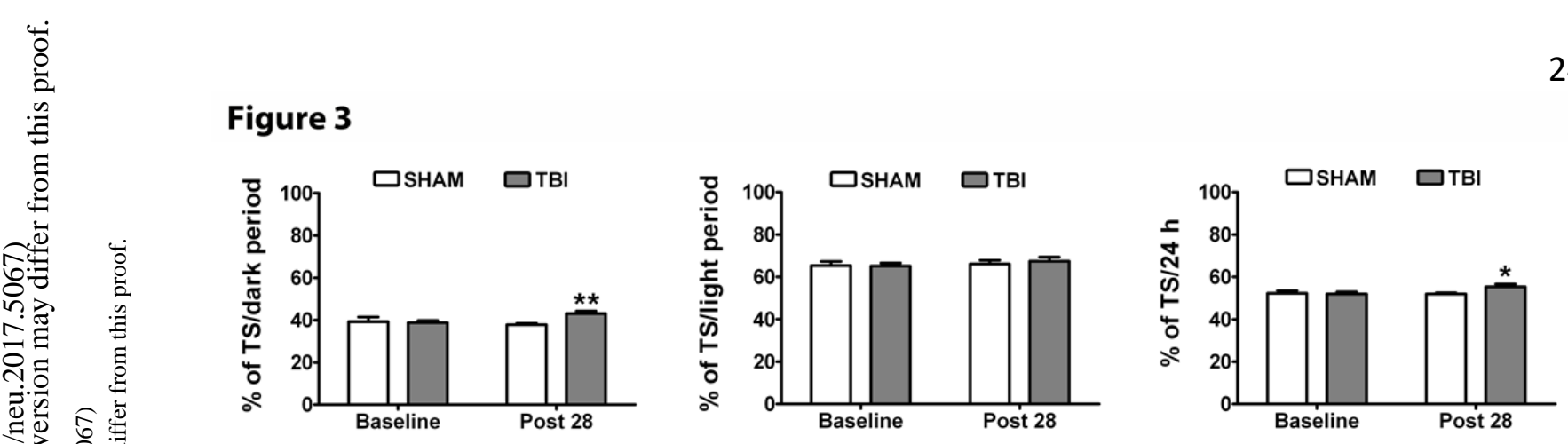

Figure 3. Sleep need 28 days after TBI. Total sleep need (TS, NREM+REM) in the dark (a), light (b) and per 24 hours (c) period at baseline and 28 days (Post 28) in SHAM or TBI rats (SHAM vs. TBI: ${ }^{*} \mathrm{P}<0.05 ;{ }^{*} \mathrm{P}<0.005$, one-tailed unpaired $\mathrm{t}$ test). $\mathrm{N}=6-7$ animals in each group, bars represent mean+/-SEM. 


\section{Figure 4}
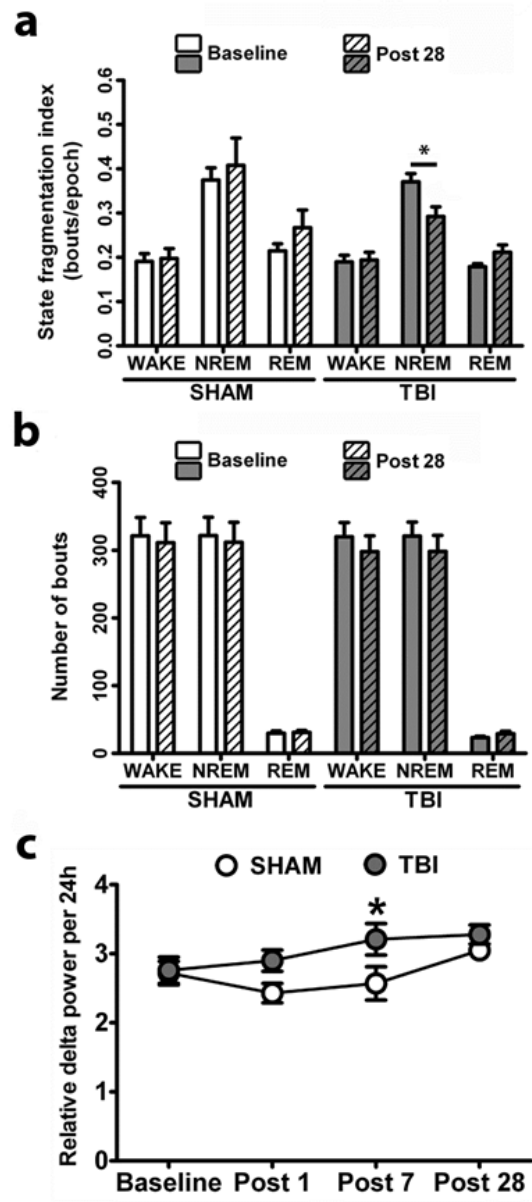

Figure 4. Behavioral state stability and relative delta power after TBI. a) State fragmentation index (bouts/epoch) in the dark period at baseline and 28 days after TBI: Decreased index for NREM sleep after TBI, BL vs. $\mathrm{P} 28$ : ${ }^{*} \mathrm{P}<0.05$, two-tailed paired $t$ test. b) Number of bouts for each state in the dark period. c) Relative delta power per 24 hours after TBI. Transient significant increase 7 days after TBI (significant effect for P7 isolated ${ }^{*} \mathrm{P}<0.05$, linear mixed model ANCOVA). $\mathrm{N}=5-7$ animals in each group, bars represent mean+/-SEM. 


\section{Figure 5}

a
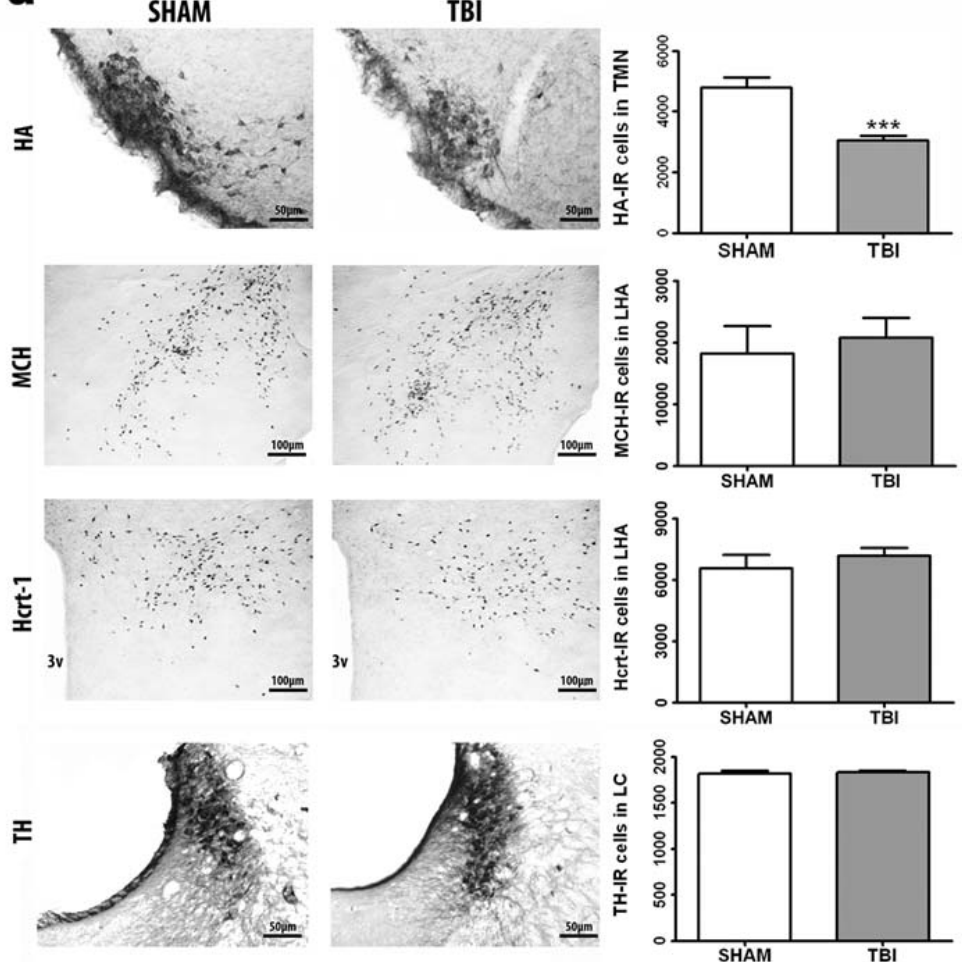

b
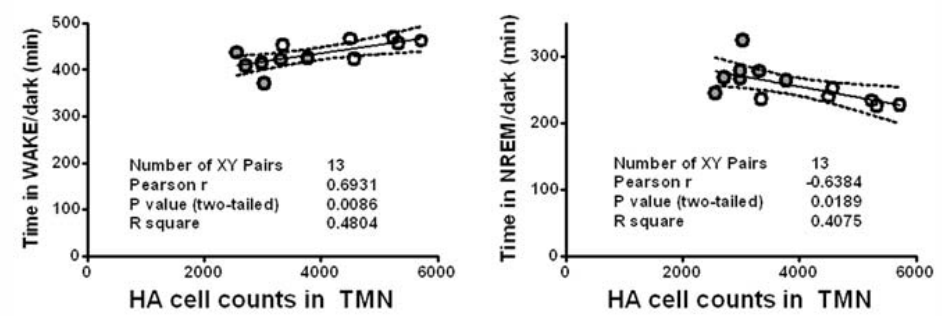

Figure 5. Immunohistochemical assessment of sleep-wake modulating neurotransmitter nuclei in hypothalamus and brainstem. a) Reduction of the number of immunoreactive histaminergic neurons by $\sim 36 \%$ in TBI animals ( $N=4-7$ brains in each group, HA: SHAM ( $n=6) 4,783 \pm 345$ vs. TBI $(n=7) 3,045 \pm 152, * * * P<0.001$, two-tailed unpaired $t$ test). HA: histamine; $\mathrm{MCH}$ : melanin-concentrating hormone, Hcrt-1: hypocretin 1, TH: tyrosin hydroxylase, TMN: tuberomammillary nucleus; LHA: lateral hypothalamic area, LC: locus coeruleus, 3v: third ventricle. Bars represent mean +/- SEM. b) Correlation plots, including best fit trend line and 95\% confidence interval, between amount of WAKE and NREM sleep in the dark period at 28 days after TBI and the number of histamine (HA) cells in the TMN (WAKE: ${ }^{*} \mathrm{P}<0.01$; NREM: ${ }^{*} \mathrm{P}<0.05$, SHAM+TBI $\mathrm{n}=13$, Pearson's correlations). 


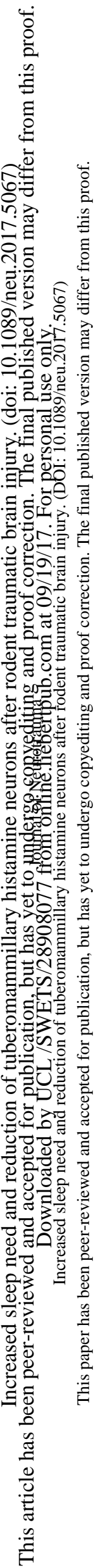

Figure 6
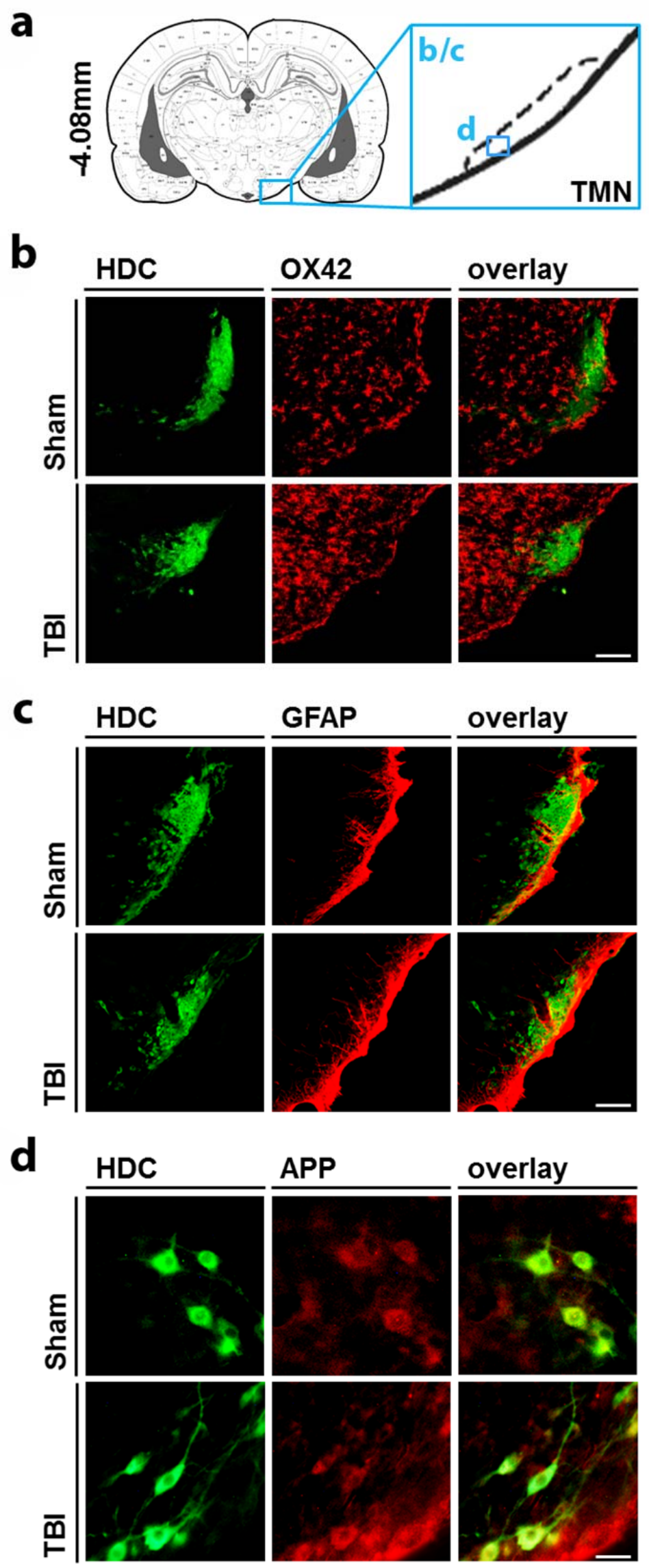
Figure 6. Immunohistochemical assessment of potential mechanisms underlying reduced histaminergic immunoreactivity. a) Representative photomicrographs of immunofluorescence stainings examining co-localization between histaminergic neurons (stained with anti-histidine decarboxylase, HDC, green) and markers of other TBI sequelae (red, middle panel) in and around the tuberomammillary nucleus (TMN). b) Enhanced neuroinflammation (stained with anti-Ox42, red) around the TMN in TBI rats as compared to SHAM controls. c) Similar levels of astrocytic scarring (stained by anti-GFAP, red) in TBI and SHAM rats. d) No salient axonal accumulation of APP-1 (red) indicating axonal injury in HDC+ TMN neurons, neither in TBI nor in SHAM rats. Scale bars in b) and c) $100 \mu \mathrm{m}$ and d) $25 \mu \mathrm{m}$. 